\title{
Sterile neutrino oscillation at a neutrino factory
}

\author{
Ken-ichi Fuki*† \\ Department of Physics, Tokyo Metropolitan University, Hachioji, Tokyo 192-0397, Japan \\ E-mail: fukiephys.metro-u.ac.jp
}

\section{Andrea Donini}

Instituto de Física Teórica UAM/CSIC, Cantoblanco, E-28049 Madrid, Spain

E-mail: andrea.donini@uam.es

\section{Davide Meloni ${ }^{\S}$}

Dipartimento di Fisica 'E. Amaldi', Università di Roma Tre

INFN, Sezione di Roma Tre, I-00146 Rome, Italy

E-mail: meloni@fis.uniroma3.it

\section{J. López-Pavón ${ }^{\mathbb{T}}$}

Departamento de Física Teórica and Instituto de Física Teórica UAM/CSIC, Universidad Autónoma de Madrid Cantoblanco, Madrid 28049, SPAIN

E-mail: jacobo.lopez@uam.es

\section{Osamu Yasuda ${ }^{\dagger}$}

Department of Physics, Tokyo Metropolitan University, Hachioji, Tokyo 192-0397, Japan

E-mail: yasuda@phys.metro-u.ac.jp

We have investigated a neutrino factory potential to look for sterile neutrinos. We point out that the golden and the silver channels are not optimal to study $(3+1)$ sterile neutrino models and the relevant channels are $v_{\mu} \rightarrow v_{\mu}$ and $v_{\mu} \rightarrow v_{\tau}$. We have studied the sensitivity to $\theta_{13}$ and to the active-sterile mixing angles $\theta_{14}, \theta_{24}$ and $\theta_{34}$ using both $v_{\mu} \rightarrow v_{\mu}$ and $v_{\mu} \rightarrow v_{\tau}$ channels at a neutrino factory. In this preliminary analysis, we have found that sensitivity limits in $\theta_{24}$ and $\theta_{34}$ are improved from the present bounds $\left(\theta_{24} \sim 12^{\circ}\right.$ and $\left.\theta_{34} \sim 30^{\circ}\right)$ to about $1^{\circ}$ and about $4^{\circ}$, respectively.

10th International Workshop on Neutrino Factories, Super beams and Beta beams June 30 - July 52008

Valencia, Spain

\footnotetext{
* Speaker.

$\dagger$ K.F. and O.Y. would like to thank the Instituto de Física Teórica UAM/CSIC for the hospitality during part of this work. This research was supported in part by the JSPS Bilateral Joint Projects (Japan-Spain).

$¥$ A.D. acknowledges funding from the Consejo Superior de Investigaciones Científicas through the bilateral japanese-spanish project 2006JP0017.

${ }^{\S}$ The work of D.M. has been partly supported the Italian Ministero dell'Universita' e della Ricerca Scientifica, under the COFIN program for 2007-08.

IJ.L.-P. acknowledges financial support from the MCIN through the FPU grant with ref. AP2005-1185.
} 


\section{Introduction}

There have been a lot of effort to interpret the LSND data in terms of neutrino oscillations. Particularly after the negative result by MiniBooNE, however, it has become diffi cult to explain the anomaly by oscillations. Despite this situation, there still remains a possibility of a sterile neutrino scenario whose mixing angles are so small that it is consistent with all the present experimental data. It is therefore worth-while to study whether the present or future neutrino experiments can investigate the existence of sterile neutrinos. The CNGS potential to look for sterile neutrinos has been investigated in [1]. We will investigate a neutrino factory potential to look for sterile neutrinos.

\section{Analytic formulae of oscillation probabilities}

We will consider a $(3+1)$ sterile neutrino model. In this model, we will make use of the following parametrization for the leptonic mixing matrix [1]:

$$
U=R_{34}\left(\theta_{34}\right) R_{24}\left(\theta_{24}\right) R_{23}\left(\theta_{23}, \delta_{3}\right) R_{14}\left(\theta_{14}\right) R_{13}\left(\theta_{13}, \delta_{2}\right) R_{12}\left(\theta_{12}, \delta_{1}\right) .
$$

The mixing angle $\theta_{13}$ and the active-sterile mixing angle $\theta_{14}$ and $\theta_{24}$ are strongly bound [1]. On the other hand, the angle $\theta_{34}$ is weakly bound. The values of $\sin \theta_{13}, \sin \theta_{14}, \sin \theta_{24}$ and $\sin ^{2} \theta_{34}$ are of the same order and we will expand the oscillation probabilities in powers of the four parameters. We only concentrate on heavy neutrinos, such that if $m_{4}$ is the mass of the neutrino eigenstate with a dominant sterile component, $\Delta m_{41}^{2} \approx \Delta m_{42}^{2} \approx \Delta m_{43}^{2} \gtrsim 0.1 \mathrm{eV}^{2}$. Neglecting $\Delta m_{\text {sol }}^{2} / \Delta m_{41}^{2}$ and $\Delta m_{\text {atm }}^{2} / \Delta m_{41}^{2}$, we get the following difference of oscillation probabilities (3+1) sterile models from the three flavor ones in the fi rst order expansion:

$$
\begin{aligned}
\delta P_{e e} & \sim \delta P_{e \mu} \sim \delta P_{e \tau} \sim \delta P_{e s} \sim O\left(s_{13}^{2}\right), \\
\delta P_{\mu \mu} & =-2\left(A_{n} L\right) s_{24} s_{34} \sin ^{2} 2 \theta_{23} \cos \delta_{3} \sin \frac{\Delta m_{a t m}^{2} L}{2 E}, \\
\delta P_{\mu \tau} & =-\sin ^{2} 2 \theta_{23} s_{34}^{2} \sin ^{2} \frac{\Delta m_{a t m}^{2}}{4 E}+\left\{s_{24} S_{34} \sin \delta_{3}+2\left(A_{n} L\right) s_{24} S_{34} \cos \delta_{3}\right\} \sin \frac{\Delta m_{a t m}^{2} L}{2 E}, \\
\delta P_{\mu s} & =\sin ^{2} 2 \theta_{23} s_{34}^{2} \sin \frac{\Delta m_{a t m}^{2} L}{4 E}-s_{24} S_{34} \sin \delta_{3} \sin \frac{\Delta m_{a t m}^{2} L}{2 E} .
\end{aligned}
$$

where $s_{i j}(i, j=1,2,3,4)$ stands for $\sin \theta_{i j}$ and $A_{n}=G_{F} N_{n} / \sqrt{2}, G_{F}$ is the Fermi constant and $N_{n}$ is the number density of neutron. Because the differences of the $\delta P_{e e}, \delta P_{e \mu}, \delta P_{e \tau}$ and $\delta P_{e s}$ in eq.(2.2) are of the second order in the small parameters, $v_{e} \rightarrow v_{x}(x=e, \mu, \tau, s)$ and $v_{x} \rightarrow v_{e}$ channels are not suitable to distinguish $(3+1)$ sterile neutrino models from three flavor ones. Thus, the golden and silver channels are not optimal to study $(3+1)$ sterile neutrino models. On the contrary, because the difference of $P_{\mu \mu}, P_{\mu \tau}$ and $P_{\mu s}$ in eq.(2.2) are of the first order, these channels are suitable to distinguish a $(3+1)$ sterile neutrino model from the three flavor one. Therefore, we will study $(3+1)$ sterile neutrino oscillation using $v_{\mu} \rightarrow v_{\mu}$ and $v_{\mu} \rightarrow v_{\tau}$ channels.

\section{Sensitivity to $(3+1)$ sterile neutrinos at a neutrino factory}

In this section, we will study the sensitivity to $\theta_{13}$ and to the active-sterile mixing angles $\theta_{14}$, $\theta_{24}$ and $\theta_{34}$ using both $v_{\mu} \rightarrow v_{\mu}$ and $v_{\mu} \rightarrow v_{\tau}$ channels at a neutrino factory. The mixing angles 
have been fi xed to $\theta_{2}=34^{\circ}$ and $\theta_{23}=45^{\circ}$. And the mass square differences have been fi xed to $\Delta m_{21}^{2}=8.0 \times 10^{-5} \mathrm{eV}^{2}$ and $\Delta m_{31}^{2}=2.4 \times 10^{-3} \mathrm{eV}^{2}$. The $\mathrm{CP}$-violating phases have been fi xed $\delta_{1}=\delta_{2}=\delta_{3}=0$ for simplicity. In this preliminary analysis, we have assumed a neutrino factory with a $50 \mathrm{GeV}$ muon beam directed at two ideal (no background and no systematic error) $50 \mathrm{kton}$ far hybrid-MIND detectors [2] at $3000 \mathrm{~km}$ and $7500 \mathrm{~km}$ away ${ }^{1}$. The number of muons in the storage ring is taken to be $2 \times 10^{20} / y r$ and we have assumed four years of running.

The first panel of figl from the left shows the sensitivity limits in $\theta_{13}$ and $\theta_{14}$. This result consistent with eq.(2.2). The second panel of fig 1 shows the sensitivity limits in $\theta_{24}$ and $\theta_{34}$. This result shows that the combination of the $v_{\mu} \rightarrow v_{\mu}$ and $v_{\mu} \rightarrow v_{\tau}$ channels has high sensitivity in both $\theta_{24}$ and $\theta_{34}$. The sensitivity limits in $\theta_{24}$ and $\theta_{34}$ are improved from the present bounds $\left(\theta_{24} \sim 12^{\circ}\right.$ and $\left.\theta_{34} \sim 30^{\circ}\right)$ to about $1^{\circ}$ and about $4^{\circ}$, respectibely. The third and the fourth panels of fig] show the sensitivity limits for $v_{\mu} \rightarrow v_{\mu}$ and $v_{\mu} \rightarrow v_{\tau}$ channels at baseline lengths $\mathrm{L}=3000 \mathrm{~km}$ and $7500 \mathrm{~km}$, respectively. These results show that the sensitivity limits in $\theta_{24}$ and $\theta_{34}$ at $3000 \mathrm{~km}$ are improved to about $1^{\circ}$ and $4^{\circ}$ at $90 \% \mathrm{CL}$ using the $v_{\mu} \rightarrow v_{\tau}$ channel, only, whereas the sensitivity limits in $\theta_{24}$ and $\theta_{34}$ at $7500 \mathrm{~km}$ are improved to about $3^{\circ}$ and $5^{\circ}$ at $90 \% \mathrm{CL}$ using the $v_{\mu} \rightarrow v_{\mu}$ channel.
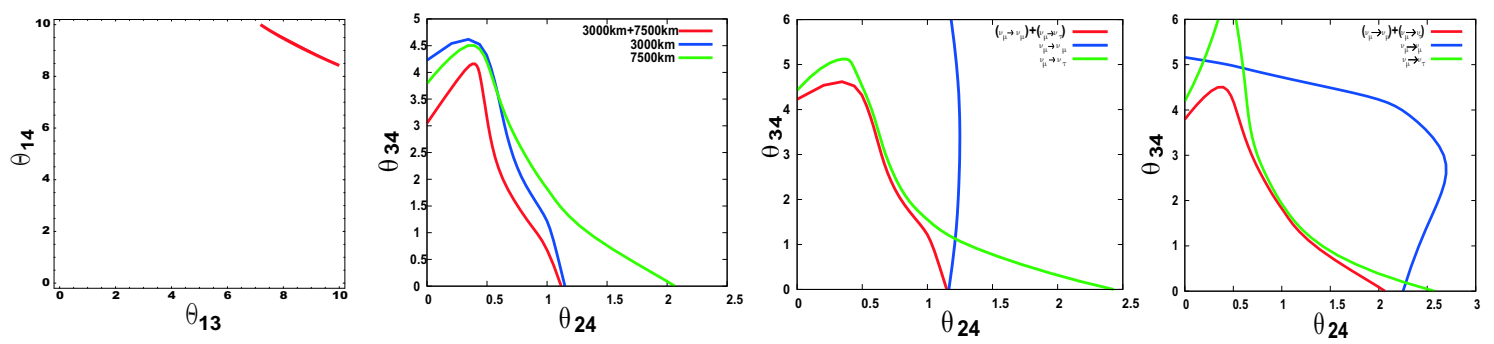

Figure 1: The first panel shows the sensitivity limit in $\theta_{3}$ and $\theta_{14}$; The second panel shows the sensitivity limits in $\theta_{24}$ and $\theta_{34}$ : the blue curve corresponds to $\mathrm{L}=3000 \mathrm{~km}$. The green curve corresponds to $\mathrm{L}=7500 \mathrm{~km}$. The red curve corresponds to a result combining $3000 \mathrm{~km}$ and $7500 \mathrm{~km}$; The third and the fourth panels show the sensitivity limits in $\theta_{24}$ and $\theta_{34}$ at $3000 \mathrm{~km}$ and $7500 \mathrm{~km}$, respectively: The blue curve corresponds to the $v_{\mu} \rightarrow v_{\mu}$ channel. The green curve corresponds to the $v_{\mu} \rightarrow v_{\tau}$ channel. The red curve corresponds to a result combining both the channels.

\section{References}

[1] A. Donini, M. Maltoni, D. Meloni, P. Migliozzi and F. Terranova, JHEP 0712, 013 (2007) [arXiv:0704.0388 [hep-ph]].

[2] A. Laing and A. Cervera, Status of MIND, in proceedings of 10th International Workshop on Neutrino Factories, Super beams and Beta beams (NuFact08), June 30 - July 5 2008, Valencia, Spain POS (NUFACT 0 8) 042 ; L. Scotto Lavina, Design and Performance of ECC-MECC, in proceedings of 10th International Workshop on Neutrino Factories, Super beams and Beta beams (NuFact08), June 30 - July 5 2008, Valencia, Spain PoS (NUFACT 08) 049.

[3] A. Donini, K. Fuki, J. Lopez-Pavon, D. Meloni and O. Yasuda, arXiv:0812.3703 [hep-ph].

\footnotetext{
${ }^{1}$ After this workshop we have improved our analysis including the backgrounds, the efficiencies and the systematic errors at a Neutrino Factory for stored muon energies $50 \mathrm{GeV}$ and $20 \mathrm{GeV}$ [3].
} 